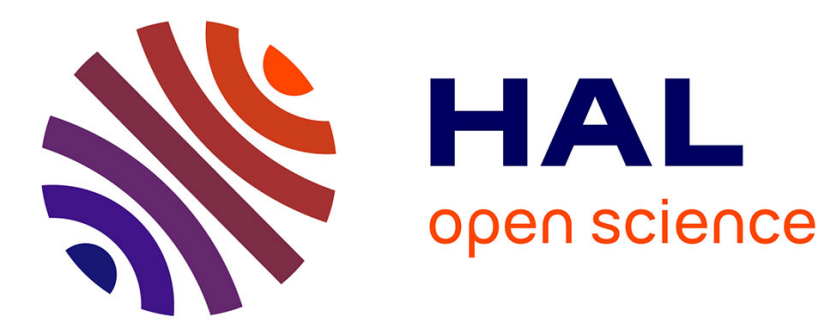

\title{
Dimension reduction for compressible pipe flows including friction \\ Mehmet Ersoy
}

\section{To cite this version:}

Mehmet Ersoy. Dimension reduction for compressible pipe flows including friction. Asymptotic Analysis, 2016, 98 (3), pp.237-255. 10.3233/ASY-161367 . hal-00908965v2

\section{HAL Id: hal-00908965}

\section{https://hal.science/hal-00908965v2}

Submitted on 15 Jun 2016

HAL is a multi-disciplinary open access archive for the deposit and dissemination of scientific research documents, whether they are published or not. The documents may come from teaching and research institutions in France or abroad, or from public or private research centers.
L'archive ouverte pluridisciplinaire HAL, est destinée au dépôt et à la diffusion de documents scientifiques de niveau recherche, publiés ou non, émanant des établissements d'enseignement et de recherche français ou étrangers, des laboratoires publics ou privés. 


\title{
Dimension reduction for compressible pipe flows including friction.
}

\author{
M. ERSOY*1 \\ ${ }^{1}$ Université de Toulon, IMATH, EA 2134, 83957 La Garde, France.
}

February 23, 2016

\begin{abstract}
We present the full derivation of a one dimensional "Saint-Venant" like equations for barotropic compressible pipe flows including friction. The one dimensional hyperbolic system is called $\gamma$-pressurized model where $\gamma$ is the adiabatic constant. It is obtained through the three dimensional barotropic NavierStokes equations under "thin layer" assumptions as a first order approximation. Prescribing suitable boundary conditions, one can introduce a general friction law and then explicitly show its geometrical (w.r.t the hydraulic radius) and hydrodynamical (w.r.t the Oser number) dependencies in the reduced model. In particular, for linear pressure law $(\gamma=1)$, we justify the one dimensional pressurized model (called $\mathbf{P}$-model) introduced by the author in the context of unsteady mixed flows in closed water pipes. For non linear pressure law $(\gamma \neq 1)$, the $\gamma$-pressurized model describes the evolution of a compressible (almost) gravityless flow.
\end{abstract}

Keywords: pressurized flow, compressible Navier-Stokes, barotropic laws, thin layer approximation, hydrostatic approximation, friction law, Oser number

AMS Subject classification : 65M08, 65M75, 76B07, 76M12, 76M28, 76N15

\section{Notations concerning geometrical quantities}

\begin{tabular}{ll}
\hline $\mathcal{P}$ & pipe \\
$\Omega(x)$ & cross-section area of the pipe orthogonal to the axis $z=Z(x)$ \\
$S(x)$ & area of $\Omega(x)$ \\
$R(x)$ & radius of the cross-section $\Omega(x)$ \\
$\theta(x)$ & angle of the inclination of the main pipe axis $z=Z(x)$ at position \\
$\sigma(x, z)$ & $x$ \\
$\varepsilon$ & width of the cross-section $\Omega(x)$ at altitude $z$ \\
\hline
\end{tabular}

\section{Notations concerning the Navier-Stokes model}

\begin{tabular}{ll}
\hline$\rho(t, x, y, z)$ & density of the fluid \\
$\mathbf{u}(t, x, y, z)=(u(t, x, y, z), \mathbf{v}(t, x, y, z))$ & velocity of the fluid \\
$p(\rho)$ & barotropic pressure law \\
$\gamma$ & adiabatic constant \\
$k(\mathbf{u})$ & border friction term \\
\hline
\end{tabular}

\footnotetext{
*Mehmet.Ersoy@univ-tln.fr
} 
Notations concerning the $\gamma$ - pressurized model

\begin{tabular}{ll}
\hline $\bar{X}(t, x)=\frac{1}{S(x)} \int_{\Omega(x)} X(t, x, y, z) d y d z$ & mean value of $X$ over $\Omega(x)$ \\
$\rho(t, x)$ & density of the averaged model \\
$u(t, x)$ & velocity of the averaged model \\
$p_{\gamma}(x, \rho)$ & averaged barotropic pressure law \\
$C$ & Oser number \\
$K_{\gamma}(x, u)$ & averaged friction \\
$s_{\gamma}(x)$ & (weighted) wet perimeter of $\Omega(x)$ \\
$\mathcal{S}_{\gamma}(x)$ & (weighted) wet area of $\Omega(x)$ \\
\hline
\end{tabular}

Bold characters are used for vectors.

\section{Introduction}

In this work, we are interested in the model reduction of a general three dimensional barotropic compressible flows occurring in thin and long closed pipes including friction. Compressible flows confined in thin pipe, often referred as pressurized flows, plays an important role in many engineering applications such as storm sewers, waste or supply pipes in hydroelectric installations, and also in the simulation of unsteady mixed flows in closed pipes, i.e., where some part of the flow can be free surface (it means that only a part of the pipe is filled) and/or pressurized (it means that the pipe is filled), see for instance $[1,2,3]$. The flow being almost unidirectional (i.e. following the main pipe axis), introducing reduced models preserving some of the main physical and geometrical features is one of the most challenging issues addressed here. Contrary to the existing literature (see for instance, $[24,21,13,3]$ ), the friction, appearing in the one dimensional reduced model, is considered and in particular we show its geometrical and hydrodynamical dependencies.

Pressurized water flows are classically modelled by the shallow water equations (see for instance $[14,12,9]$ or [18]), commonly used to describe free surface flows in open channels, with the artifice of Preissman (see for example $[7,6,23]$ ) assuming a narrow slot to exist in the upper part of the pipe. The width of the slot is calibrated to provide the correct sonic speed. Nevertheless, as pointed out by several authors (see [20] for instance), the pressurizing phenomenon is a dynamic shock requiring a full dynamic treatment even if the inflows and other boundary conditions change slowly [15]. Moreover, the Preissman slot artefact is unable to take into account the depressurisation phenomenon (i.e. sub-atmospheric flows) which occurs during a "water-hammer". A model for pressurized flow, called $\mathbf{P}$-model, has been proposed by Bourdarias et al $[1,2,3]$ to take into account sub-atmospheric flows. For this purpose, the fluid is assumed to be compressible and governed by the three dimensional barotropic Euler equations with a linearized pressure pressure law. Then, through a formal model reduction by section averaging, assuming the well-known "motion by slices" property, they obtain the frictionless one dimensional $\mathbf{P}$-model. In order to deal with the pipe friction, a nonlinear damping term is added.

Unlike the previous works, we propose here to study the full derivation of a one dimensional pressurized flows, including the friction, from the three dimensional barotropic Navier-Stokes equations. Furthermore, we generalize the model reduction to non linear pressure law $p(\rho)=\alpha \rho^{\gamma}$ where $\gamma$ and $\alpha$ are some positive constants. The reduced system, named $\gamma$-pressurized model, is a first order hyperbolic model. In comparison with the work by Bourdarias et al [3], assuming viscous instead of inviscid flows allows, first, to consider through a wall-law condition the border friction and, second, to get the "motion by slices" property required in the model reduction. In particular, we are able to justify and to show the geometrical and hydrodynamical dependencies of the "section-averaged" friction commonly used by the engineers community. More precisely, we show the role of the gravity force through the Oser number (defined as $M_{a} / F_{r}$ where $M_{a}$ stands for the Mach number and $F_{r}$ is the Froude number) and the hydraulic radius to any pipe section. Let us recall that the hydraulic radius is a length scale for non-circular ducts introduced to use the analysis done for circular pipes (see for instance [21, 22]). Let us also emphasize that for non-linear pressure law, i.e. $\gamma \neq 1$, the $\gamma$-pressurized models are characterized by a low Oser number, i.e. relevant almost for gravityless flows. 
In particular, in this work, we provide a justification of the full model (including the friction) introduced by Bourdarias et al [3] for a linear pressure law and the air entrainment model (including the friction) by Bourdarias et al [4] for non linear pressure law. Using the same process, in [9], we propose a one dimensional Saint-Venant equations including friction from the three dimensional incompressible Navier-Stokes equations. Let us also mention that Morales [18] proposes also a model for shallow water flows but composed of two layers of compressible-incompressible fluids. This model is obtained through the model reduction of the compressible and incompressible Euler equations as superposed layers.

The paper is organized as follows. In Section 2, we recall the full compressible Navier-Stokes equations and we define the boundary conditions including a general border friction law. The "motion by slices" property under large Reynolds flows is obtained through the hydrostatic approximation in Section 3. Next, under suitable assumptions, these equations are averaged through the pipe section assumed to be orthogonal to the main flow direction. Then, we obtain the $\gamma$-pressurized model and we complete the presentation, in Section 4 , with a numerical illustration of the influence of the Oser number on the model.

\section{The compressible Navier-Stokes equations and its closure}

In this section, we fix the notations of the geometrical quantities involved to describe the thin domain representing a pipe. In particular, without loss of generality (see Remark 2.1), we consider the case of pipe with circular section.

\subsection{Geometrical settings}

Let us consider a compressible fluid confined in a three dimensional rigid domain $\mathcal{P}$ representing, a pipe of length $L$ :

$$
\mathcal{P}:=\left\{(x, y, z) \in \mathbb{R}^{3} ; x \in[0, L],(y, z) \in \Omega(x)\right\}
$$

where the section $\Omega(x), x \in[0, L]$, is

$$
\Omega(x)=\left\{(y, z) \in \mathbb{R}^{2} ; y \in[\alpha(x, z), \beta(x, z)], z \in[-R(x), R(x)]\right\}
$$

as displayed in figure 1(a). Flows are assumed to be oriented in the i-direction of the pipe.

The section $\Omega(x), x \in[0, L]$, is assumed to be orthogonal to the main flow direction. Here $R(x)$ stands for the radius of the pipe section $S(x)=\pi R^{2}(x), \alpha(x, z)$ (resp. $\beta(x, z)$ ) is the left (resp. the right) boundary point at elevation $-R(x) \leqslant z \leqslant R(x)$. In the $\Omega$-plane, we define the coordinate of a point $\mathbf{m} \in \partial \Omega(x)$, $x \in[0, L]$, by $(y, \varphi(x, y))$ where $\varphi(x, y)=\sqrt{R(x)^{2}-y^{2}}$ for $y>0$ and $\varphi(x, y)=-\sqrt{R(x)^{2}-y^{2}}$ for $y<0$. The point $\mathbf{m}$ stands for the vector $\omega \mathbf{m}$ where $w(x, 0, Z(x))$ defines the main slope elevation of the pipe with $\frac{d Z}{d x}(x)=-\sin \theta(x)$. We note $\mathbf{n}=\frac{\mathbf{m}}{|\mathbf{m}|}$ the outward unit vector at the point $\mathbf{m} \in \partial \Omega(x), x \in[0, L]$ as represented in figure $1(\mathrm{~b})$.

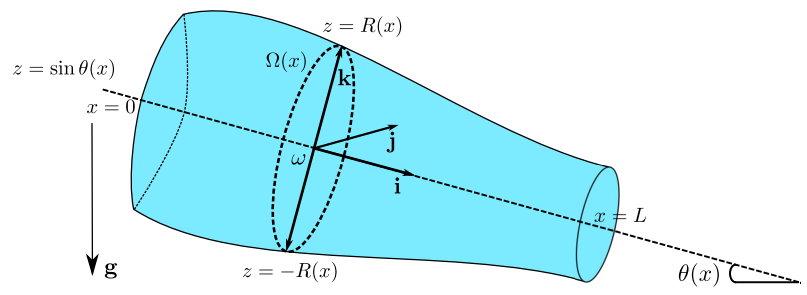

(a) Configuration

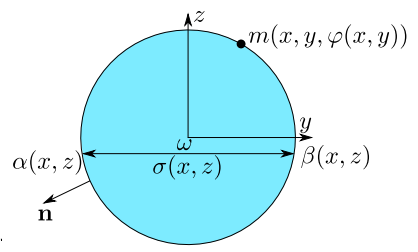

(b) $\Omega$-plane

Figure 1: Geometric characteristics of the pipe. 
Remark 2.1. One can easily adapt this work to any realistic pipe. For instance in the case of "horseshoe" section (see figure 2.1), the section $\Omega(x), x \in[0, L]$, is given by

$$
\Omega(x)=\Omega_{H}(x) \cup \Omega_{R}(x)
$$

where

$$
\Omega_{H}(x)=\left\{(y, z) \in \mathbb{R}^{2} ; y \in[\alpha(x, z), \beta(x, z)], z \in[0, H(x)]\right\}
$$

and

$$
\Omega_{R}(x)=\left\{(y, z) \in \mathbb{R}^{2} ; y \in[\alpha(x, z), \beta(x, z)], z \in[H(x), R(x)]\right\} .
$$

$H$ is the height of the trapezoidal basis and $R$ is the radius of the upper part of the "horseshoe".

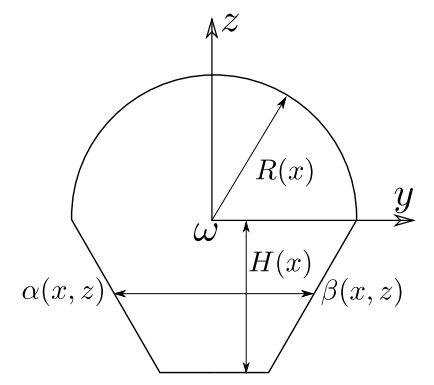

Figure 2: A pipe with a "horseshoe" section

\subsection{The compressible Navier-Stokes model}

In the domain $\mathcal{P}$, we assume that the pipe is completely filled with a viscous barotropic and compressible fluid. The pressure $p=p(\rho)$ is thus a linear or non linear function of the fluid density $\rho$. The inner wall of the pipe is assumed non smooth. Thus, we consider the three dimensional barotropic compressible Navier-Stokes equations with a prescribed general wall law conditions including friction. The system is then completed with inflows and outflows conditions at the upstream and downstream ends.

The governing equations for the motion of a barotropic compressible fluid in $[0, T] \times \mathcal{P}, T>0$ are

$$
\left\{\begin{aligned}
\partial_{t} \rho+\operatorname{div}(\rho \mathbf{u}) & =0 \\
\partial_{t}(\rho \mathbf{u})+\operatorname{div}(\rho \mathbf{u} \otimes \mathbf{u})-\operatorname{div} \sigma & =\rho F \\
p & =p(\rho)
\end{aligned}\right.
$$

where $\mathbf{u}=\left(\begin{array}{c}u \\ \mathbf{v}\end{array}\right)$ is the velocity fields with $u$ the $\mathbf{i}$-component and $\mathbf{v}=\left(\begin{array}{c}v \\ w\end{array}\right)$ the $\Omega$-component. The right hand side $F$ represents the external gravity force of constant $g$

$$
F=g\left(\begin{array}{c}
\sin \theta(x) \\
0 \\
-\cos \theta(x)
\end{array}\right) \text {. }
$$

The total Cauchy stress tensor reads

$$
\sigma=\left(\begin{array}{cc}
-p+\lambda \operatorname{div}(\mathbf{u})+2 \mu \partial_{x} u & \mathcal{R}(\mathbf{u})^{t} \\
\mathcal{R}(\mathbf{u}) & -p I_{2}+\lambda \operatorname{div}(\mathbf{u}) I_{2}+2 \mu D_{y, z}(\mathbf{v})
\end{array}\right)
$$

where $I_{2}$ is the identity matrix, $\mu$ is the dynamical viscosity and $\mathcal{R}(\mathbf{u})=\mu\left(\nabla_{y, z} u+\partial_{x} \mathbf{v}\right)$. The quantity $\nabla_{y, z} u=\left(\begin{array}{c}\partial_{y} u \\ \partial_{z} u\end{array}\right)$ is the gradient of $u$ with respect to $(y, z)$. Noting $X^{t}$ the transpose of $X$, we define the strain tensor $D_{y, z}(\mathbf{v})$ with respect to the variable $(y, z)$ :

$$
2 D_{y, z}(\mathbf{u})=\nabla_{y, z} \mathbf{v}+\nabla_{y, z}^{t} \mathbf{v} .
$$


Finally, the pressure law is given by the following equation of state:

$$
p(\rho)=\alpha \rho^{\gamma}
$$

where $\alpha$ is a positive physical constant.

Let us end this section with several comments:

\section{Remark 2.2.}

1. The last term $\lambda \operatorname{div}(\mathbf{u})$ in (3) is the classical normal stress tensor which plays an important role when the fluid is rapidly compressed or expanded as in shock waves. The quantity $\lambda$ is called the volume viscosity, often called second viscosity, and it is usually assumed to be of the same order of the dynamical viscosity $\mu$ for the applications under considerations (see, for instance, [17]).

2. To force a privileged flow direction, the total Cauchy stress tensor can be also defined as follows (see, for instance, Ersoy et al $[11,10])$

$$
\sigma=-p I_{3}+2 \Sigma \cdot D(u)+\lambda \operatorname{div}(u) I_{3} .
$$

Through the term

$$
\Sigma . D(u)=\left(\begin{array}{cc}
2 \mu D_{x}(\mathbf{u}) & \mu_{2} \mathcal{R}(\mathbf{u})^{t} \\
\mu_{3} \mathcal{R}(\mathbf{u}) & 2 \mu_{3} \partial_{y} v
\end{array}\right)
$$

with the following anisotropic viscous tensor:

$$
\Sigma=\Sigma(t, x, y)\left(\begin{array}{ccc}
\mu(t, x, y) & \mu(t, x, y) & \mu_{2}(t, x, y) \\
\mu(t, x, y) & \mu(t, x, y) & \mu_{2}(t, x, y) \\
\mu_{3}(t, x, y) & \mu_{3}(t, x, y) & \mu_{3}(t, x, y)
\end{array}\right)
$$

the horizontal (i.e. with respect to $x$ ) can be set as the privileged flow direction. The anisotropic property is useful for compressible flows as in the context of the atmosphere modelling [16].

3. With the equations above, pressurized water pipe flows (see [3] for further details) can be described by setting $\gamma=1$ with $\sqrt{\alpha}=c$ in the equation of state (4). The quantity $c$ is the sonic speed defined by $c=\frac{1}{\sqrt{\beta_{0} \rho_{0}}} \approx 1400 \mathrm{~m}^{2} / \mathrm{s}$ where $\beta_{0} \approx 5.010^{-10} \mathrm{~m}^{2} / N$ stands for the inverse of the bulk modulus of compression of the water and $\rho_{0}$ is the volumetric mass of water. As described in Section 1, effects such as overpressure (sup-atmospheric) or depression (sub-atmospheric) state can be modelled with the following the equation of state

$$
p(\rho)=p_{a}+c^{2}\left(\rho-\rho_{0}\right) .
$$

This formula has the advantage to show clearly those states. An overpressure (respectively depression) state is observed if $\rho>\rho_{0}$ (respectively $\rho<\rho_{0}$ ).

4. With the above equations, pressurized air pipe flows can be modelled by setting the adiabatic index $\gamma$ to $\frac{7}{5}$ and $\sqrt{\alpha}=\frac{p_{a}}{\rho_{a}^{\gamma}}$ for some reference pressure $p_{a}$ and density $\rho_{a}$ (see for instance [4]).

\subsection{The boundary conditions}

For pipe flow calculations, the Darcy-Weisbach equation is generally adopted which is valid for laminar as well as for turbulent flows. Roughly speaking, such formula relates losses $h$ occurred during flows and it reads:

$$
h=C_{f} \frac{L}{D} \frac{U^{2}}{2 g}
$$

where $L, D, U$ are the pipe length, the pipe diameter and the velocity. The friction factor $C_{f}$, instead of being a simple constant, turns out to be a factor that depends upon several parameters such as the 
Reynolds number $R_{e}$, the relative roughness $\delta$ of the material, the Froude number $F_{r}$, the Mach number $M_{a}$, geometrical parameters, ...

Following the type of the material, rough or smooth pipe, leaves $C_{f}$ depend upon less quantities and lead to several expressions. An empirical transition function for the region between smooth pipes and the complete turbulence zone has been proposed by Colebrook:

$$
\frac{1}{\sqrt{C_{f}}}=-0.86 \ln \left(\frac{\delta}{3.7 D}+\frac{2.51}{R_{e} \sqrt{C_{f}}}\right) .
$$

Because of the extreme complexity of the rough surfaces, most of the advances in the comprehension have been developed around experiments leading to charts such as the Moody-Stanton diagram, expressing $C_{f}$ as a function of the Reynolds number, the relative roughness and some geometrical parameters depending on the material. This yields to several formula depending on the modelling, for instance Chézy and Manning which are well-known by the engineers community, see for instance [21, 23] or [3].

For laminar flow, the effects of the material roughness can be ignored due to a presence of a thin laminar film at the pipe wall. Then, it can be shown that the Darcy-Weisbach equation reduces to $C_{f}=\frac{64}{R_{e}}$ that we note $C_{f}=C_{l}$ in the sequel. And, the losses are directly proportional to the velocity. When increasing the Reynolds number, the thin laminar film becomes unstable and causes turbulence increasing the head loss. Thus, the dependency to the Reynolds number can be neglected and the head loss is almost directly proportional to $U^{2}$. The value of the friction factor, that we note $C_{f}=C_{t}$ in the sequel, can be read on diagrams.

In particular, this motivates the use of the following general friction law:

$$
k(\mathbf{u}) \mathbf{u}=C_{f}(|\mathbf{u}|) \mathbf{u}=C_{l} \mathbf{u}+C_{t}|\mathbf{u}| \mathbf{u}, C_{l} \geqslant 0, C_{t}>0 .
$$

We do not intend in this work to define precisely the friction law but instead of, we want to directly include it in its general form to explicitly show its dependency on physical parameters in the present model reduction (for specific applications, we refer to [8])

Thus, on the inner wall $\partial \Omega(x), \forall x \in(0, L)$, we assume a wall-law condition including a general friction law:

$$
\left(\sigma(\mathbf{u}) \mathbf{n}_{b}\right) \cdot \tau_{b_{i}}=\rho k(\mathbf{u}) \mathbf{u} \cdot \tau_{b_{i}}, \quad x \in(0, L),(y, z) \in \Gamma_{b}(x), i=1,2
$$

where $\tau_{b_{i}}$ is the $i^{\text {th }}$ vector of the tangential basis and $\mathbf{n}_{b}$ stands for the unit outward normal vector:

$$
\mathbf{n}_{b}=\frac{1}{\sqrt{\left(\partial_{x} \varphi\right)^{2}+\mathbf{n} \cdot \mathbf{n}}}\left(\begin{array}{c}
-\partial_{x} \varphi \\
\mathbf{n}
\end{array}\right)
$$

with $\mathbf{n}=\left(\begin{array}{c}-\partial_{y} \varphi \\ 1\end{array}\right)$ the outward normal vector in the $\Omega$-plane. Writing the wall-law condition in its vectorial form (i.e. the tangential constraints),

$$
\sigma(\mathbf{u}) \mathbf{n}_{b}-\left(\sigma(\mathbf{u}) \mathbf{n}_{b} \cdot \mathbf{n}_{b}\right) \mathbf{n}_{b}=\rho k(\mathbf{u}) \mathbf{u}, \quad t>0, x \in(0, L),(y, z) \in \Gamma_{b}(t, x),
$$

one can split up the $\mathbf{i}$-component and the $(\mathbf{j}, \mathbf{k})$-components. Thus, the wall-law boundary conditions are

$$
\begin{array}{r}
\mathcal{R}(\mathbf{u}) \cdot \mathbf{n}\left(\mathbf{n} \cdot \mathbf{n}-\left(\partial_{x} \varphi\right)^{2}\right)+2 \mu \partial_{x} \varphi\left(D_{y, z}(\mathbf{v}) \mathbf{n} \cdot \mathbf{n}-\partial_{x} u(\mathbf{n} \cdot \mathbf{n})\right)=\left(\mathbf{n} \cdot \mathbf{n}+\left(\partial_{x} \varphi\right)^{2}\right)^{3 / 2} \rho k(u) u \\
2 \mu\left(\partial_{x} \varphi\right)^{2}\left(D_{y, z}(\mathbf{v}) \mathbf{n}-\mathbf{n}\right)+\partial_{x} \varphi \mathcal{R}(\mathbf{u})\left(\mathbf{n} \cdot \mathbf{n}-\left(\partial_{x} \varphi\right)^{2}\right)=\left(\mathbf{n} \cdot \mathbf{n}+\left(\partial_{x} \varphi\right)^{2}\right)^{3 / 2} \rho k(\mathbf{v}) \mathbf{v}
\end{array}
$$

These equations are supplemented with a no-penetration condition:

$$
\mathbf{u} \cdot \mathbf{n}_{b}=0, \quad t>0, x \in(0, L),(y, z) \in \Gamma_{b}(t, x)
$$

i.e.

$$
u \partial_{x} \varphi=\mathbf{v} \cdot \mathbf{n}, \quad t>0, x \in(0, L),(y, z) \in \Gamma_{b}(t, x)
$$




\section{The averaged model}

The technique presented in this section is inspired by the work by Gerbeau and Perthame [14] in the context of the reduction of the two dimensional incompressible Navier-Stokes model to the one dimensional Saint-Venant equations. In this paper, we proceed to the reduction of the three-dimensional compressible Navier-Stokes equations to a one dimensional shallow water like equations taking into account a general friction law.

\subsection{Dimensionless Navier-Stokes equations}

In the sequel we consider the non-dimensional form of the Navier-Stokes system using the shallow water assumption by introducing a "small" parameter such that

$$
\varepsilon=\frac{D}{L}=\frac{W}{U}=\frac{V}{U} \ll 1
$$

where $U, \boldsymbol{V}=(V, W)$ are the characteristic speeds in the $\mathbf{i}$-direction and the $(\mathbf{j}, \mathbf{k})$-direction.

We introduce a characteristic time $T$ such that $T=\frac{L}{U}$. The dimensionless quantities of time $\tilde{t}$, coordinate $(\tilde{x}, \tilde{y}, \tilde{z})$ and velocity field $(\tilde{u}, \tilde{v}, \tilde{w})$, noted temporarily by a $\tilde{*}$, are defined by

$$
\tilde{t}=\frac{t}{T}, \quad(\tilde{x}, \tilde{y}, \tilde{z})=\left(\frac{x}{L}, \frac{y}{D}, \frac{z}{D}\right), \quad(\tilde{u}, \tilde{v}, \tilde{w})=\left(\frac{u}{U}, \frac{v}{W}, \frac{w}{W}\right), \quad \tilde{\rho}=\frac{\rho}{r}
$$

where $r$ is a characteristic density. We also consider the modified friction factor $C_{f} / U$ that we write in the sequel $C_{f}$.

Let us define the following non-dimensional numbers:

$$
\begin{array}{llll}
F_{r} & \text { Froude number following the } \Omega \text {-plane } & : & F_{r}=U / \sqrt{g D}, \\
F_{L} & \text { Froude number following the i-direction } & : & F_{L}=U / \sqrt{g L}, \\
R_{\mu} & \text { Reynolds numbers with respect to } \mu & : & R_{\mu}=\rho_{0} U L / \mu, \\
R_{\lambda} & \text { Reynolds numbers with respect to } \lambda & : & R_{\lambda}=\rho_{0} U L / \lambda, \\
M_{a} & \text { Mach number } & : & M_{a}=U / \sqrt{\alpha}, \\
C & \text { Oser number } & : C=M_{a} / F_{r}=\sqrt{g D} / \sqrt{\alpha} .
\end{array}
$$

Using these new variables in Equations (1), dropping the $\tilde{r}$, ordering the terms with respect to $\varepsilon$, the dimensionless compressible Navier-Stokes system becomes:

$$
\begin{aligned}
\partial_{t} \rho+\partial_{x}(\rho u)+\operatorname{div}_{y, z}(\rho \mathbf{v}) & =0 \\
\partial_{t}(\rho u)+\partial_{x}\left(\rho u^{2}\right)+\operatorname{div}_{y, z}(\rho u \mathbf{v})+\frac{1}{M_{a}^{2}} \partial_{x} \rho^{\gamma} & =\rho \frac{\sin \theta(x)}{F_{L}^{2}}+\operatorname{div}_{y, z}\left(\frac{R_{\mu}^{-1}}{\varepsilon^{2}} \nabla_{y, z} u\right)+R_{\varepsilon, 1}(\mathbf{u}), \\
\frac{1}{M_{a}^{2}} \nabla_{y, z} \rho^{\gamma} & =\left(\begin{array}{c}
0 \\
-\frac{\rho \cos \theta(x)}{F_{r}^{2}}
\end{array}\right)+R_{\varepsilon, 2}(\mathbf{u}),
\end{aligned}
$$

where

and

$$
R_{\varepsilon, 1}(\mathbf{u})=R_{\mu}^{-1}\left(\partial_{x}\left(2 \partial_{x} u+\frac{R_{\lambda}^{-1}}{R_{\mu}^{-1}} \operatorname{div}(\mathbf{u})\right)+\operatorname{div}_{y, z}\left(\partial_{x} \mathbf{v}\right)\right)=O\left(R_{\mu}^{-1}\right)
$$

$$
\begin{aligned}
R_{\varepsilon, 2}(\mathbf{u})= & R_{\mu}^{-1}\left(\partial_{x}\left(\nabla_{y, z} u+\varepsilon^{2} \partial_{x} \mathbf{v}\right)+\operatorname{div}_{y, z}\left(\frac{R_{\lambda}^{-1}}{R_{\mu}^{-1}} \operatorname{div}(\mathbf{u})+2 D_{y, z}(\mathbf{v})\right)\right) \\
& -\varepsilon^{2}\left(\partial_{t}(\rho \mathbf{v})+\partial_{x}(\rho u \mathbf{v})+\operatorname{div}_{y, z}(\rho \mathbf{v} \otimes \mathbf{v})\right) \\
= & R_{\mu}^{-1}\left(\partial_{x}\left(\nabla_{y, z} u\right)+\operatorname{div}_{y, z}\left(\frac{R_{\lambda}^{-1}}{R_{\mu}^{-1}} \operatorname{div}(\mathbf{u})+2 D_{y, z}(\mathbf{v})\right)\right)+\boldsymbol{O}\left(\varepsilon^{2}\right) \\
= & \boldsymbol{O}\left(R_{\mu}^{-1}\right)+\boldsymbol{O}\left(\varepsilon^{2}\right) .
\end{aligned}
$$


The first component of the wall-law boundary condition (6) becomes:

$$
\begin{aligned}
\frac{R_{\mu}^{-1}}{\varepsilon} \nabla_{y, z} u \cdot \mathbf{n} & =\frac{\left(\mathbf{n} \cdot \mathbf{n}+\varepsilon^{2}\left(\partial_{x} \varphi\right)^{2}\right)^{3 / 2} \rho k(u) u}{\left(\mathbf{n} \cdot \mathbf{n}-\varepsilon^{2}\left(\partial_{x} \varphi\right)^{2}\right)}-\varepsilon R_{\mu}^{-1}\left(\frac{2 \partial_{x} \varphi\left(D_{y, z}(\mathbf{v}) \mathbf{n} \cdot \mathbf{n}-\partial_{x} u(\mathbf{n} \cdot \mathbf{n})\right)}{\left(\mathbf{n} \cdot \mathbf{n}-\varepsilon^{2}\left(\partial_{x} \varphi\right)^{2}\right)}+\partial_{x} \mathbf{v} \cdot \mathbf{n}\right) \\
& =\rho \sqrt{\mathbf{n} \cdot \mathbf{n}} k(u) u+O\left(\varepsilon^{2}\right)+O\left(\varepsilon R_{\mu}^{-1}\right) \\
& =\rho K(u)+O\left(\varepsilon^{2}\right)+O\left(\varepsilon R_{\mu}^{-1}\right)
\end{aligned}
$$

where we make use of the notations

$$
K(u)=\sqrt{\mathbf{n} \cdot \mathbf{n}} k(u) u \quad \text { and } \quad \nabla_{y, z} u \cdot \mathbf{n}:=\partial_{\mathbf{n}} u
$$

which are respectively the friction term and the normal derivative of $u$ in the $\Omega$-plane.

The second component of the wall-law boundary condition (7) becomes:

$$
\begin{aligned}
R_{\mu}^{-1} \nabla_{y, z} u & =\frac{\varepsilon^{2}\left(\mathbf{n} \cdot \mathbf{n}+\varepsilon^{2}\left(\partial_{x} \varphi\right)^{2}\right)^{3 / 2} \rho k(\mathbf{v}) \mathbf{v}}{\partial_{x} \varphi\left(\mathbf{n} \cdot \mathbf{n}-\varepsilon^{2}\left(\partial_{x} \varphi\right)^{2}\right)}-\frac{2 \varepsilon^{3} R_{\mu}^{-1} \partial_{x} \varphi^{2}\left(D_{y, z}(\mathbf{v}) \mathbf{n}-\mathbf{n}\right)}{\partial_{x} \varphi\left(\mathbf{n} \cdot \mathbf{n}-\varepsilon^{2}\left(\partial_{x} \varphi\right)^{2}\right)}-\varepsilon^{2} \partial_{x} \mathbf{v} \cdot \mathbf{n} \\
& =\boldsymbol{O}\left(\varepsilon^{2}\right)+\boldsymbol{O}\left(\varepsilon^{3} R_{\mu}^{-1}\right)
\end{aligned}
$$

\subsection{First order approximation}

As emphasized before in Section 2.3 and Remark 2.2, when increasing the Reynolds number $R_{\mu}$ and $R_{\lambda}$, we observe instabilities at the pipe wall leading to turbulent flows. Assuming the characteristic length of the thin unstable film larger than the relative roughness of the pipe, one can always assume smallness assumption of the friction factor (see for instance [21, 19, 23]). In particular, it motivates for large Reynolds number, the following asymptotic assumptions:

$$
R_{\lambda}^{-1}=\varepsilon \lambda_{0}, \quad R_{\mu}^{-1}=\varepsilon \mu_{0}, \quad K=\varepsilon K_{0}
$$

where $\lambda_{0}, \mu_{0}$ are some constant viscosity and $K_{0}$ is the asymptotic friction law

$$
K_{0}(u)=k(u) u .
$$

Under these assumptions, the Archimedes principle is applicable and induces small vertical accelerations. As a consequence, one can drop all terms of order $O\left(\varepsilon^{2}\right)$ in Equations (9)-(11). Then, taking the formal limit as $\varepsilon$ vanishes, we deduce the hydrostatic equations (or the compressible primitive equations, see for instance $[16,11,10])$

$$
\begin{aligned}
\partial_{t} \rho_{\varepsilon}+\partial_{x}\left(\rho_{\varepsilon} u_{\varepsilon}\right)+\operatorname{div}_{y, z}\left(\rho_{\varepsilon} \boldsymbol{v}_{\varepsilon}\right) & =0 \\
\partial_{t}\left(\rho_{\varepsilon} u_{\varepsilon}\right)+\partial_{x}\left(\rho_{\varepsilon} u_{\varepsilon}^{2}\right)+\operatorname{div}_{y, z}\left(\rho_{\varepsilon} u_{\varepsilon} \boldsymbol{v}_{\varepsilon}\right)+\frac{1}{M_{a}^{2}} \partial_{x} \rho_{\varepsilon}^{\gamma} & =\rho_{\varepsilon} \frac{\sin \theta(x)}{F_{L}^{2}}+\operatorname{div}_{y, z}\left(\frac{\mu_{0}}{\varepsilon} \nabla_{y, z} u_{\varepsilon}\right), \\
\frac{1}{M_{a}^{2}} \nabla_{y, z} \rho_{\varepsilon}^{\gamma} & =\left(\begin{array}{c}
0 \\
-\frac{\rho_{\varepsilon} \cos \theta(x)}{F_{r}^{2}}
\end{array}\right) .
\end{aligned}
$$

Let us emphasize that even if this system results from a formal limit, we prefer to note its solution $\left(\rho_{\varepsilon}, u_{\varepsilon}, \boldsymbol{v}_{\varepsilon}\right)$

due to the explicit dependency to $\varepsilon$ through the term $\operatorname{div}_{y, z}\left(\frac{\mu_{0}}{\varepsilon} \nabla_{y, z} u_{\varepsilon}\right)$ in Equation (17). At zeroth order, this term is precisely the friction at the inner pipe wall. Indeed, the boundary conditions (12) and (13) provide at zeroth order

$$
\frac{\mu_{0}}{\varepsilon} \nabla_{y, z} u_{\varepsilon} \cdot \mathbf{n}=\rho_{\varepsilon} K_{0}\left(u_{\varepsilon}\right) \quad \text { and } \quad \mu_{0} \nabla_{y, z} u_{\varepsilon}=0, \quad x \in(0, L),(y, z) \in \partial \Omega(x) .
$$


Moreover, identifying terms at order $\frac{1}{\varepsilon}$ in Equations (16)-(18), we obtain the so-called "motion by slices"

$$
u_{\varepsilon}(t, x, y, z)=u_{0}(t, x)+O(\varepsilon)
$$

for some function $u_{0}(t, x)$, by solving the Neumann problem, for $t>0, x \in(0, L)$ :

$$
\left\{\begin{array}{lll}
\operatorname{div}_{y, z}\left(\mu_{0} \nabla_{y, z} u_{\varepsilon}\right) & =O(\varepsilon), \quad(y, z) \in \Omega(x) \\
\mu_{0} \partial_{\mathbf{n}} u_{\varepsilon} & =O(\varepsilon), \quad(y, z) \in \partial \Omega(x)
\end{array} .\right.
$$

As a consequence, the following approximation holds at first order

$$
\left.u_{\varepsilon}(t, x, y, z) \approx \overline{u_{\varepsilon}} t, x\right)
$$

where

$$
\left.\overline{u_{\varepsilon}} t, x\right)=\frac{1}{S(x)} \int_{\Omega(x)} u_{\varepsilon}(t, x, y, z) d y d z
$$

stands for the mean value of the fluid velocity over $\Omega(x)$ of area $S(x)$ :

$$
S(x)=\int_{\Omega(t, x)} d y d z=\int_{-R(x)}^{R(x)} \sigma(x, z) d z .
$$

Therefore, one can approximate the non-linear term $u_{\varepsilon}^{2}$ in Equation (17) by

$$
\overline{u_{\varepsilon}^{2}} \approx \bar{u}_{\varepsilon}^{2} \text {. }
$$

Furthermore, using the Equations (18), keeping in mind the equation of state (4), we get

$$
\rho_{\varepsilon}(t, x, y, z)= \begin{cases}\rho_{0}(t, x) \exp \left(-C^{2} \cos \theta(x) z\right)+O(\varepsilon) & \text { if } \quad \gamma=1 \\ \rho_{0}(t, x) N(t, x, y, z)+O(\varepsilon) & \text { if } \quad \gamma \neq 1\end{cases}
$$

with

$$
N(t, x, z)=\left(1+z C^{2} \cos \theta(x) \frac{1-\gamma}{\gamma \rho_{0}(t, x)^{\gamma-1}}\right)^{\frac{1}{\gamma-1}}
$$

for some positive function $\rho_{0}$, called density.

Remark 3.1. For $\gamma=1$, Equations (16)-(18) are the so-called Compressible Primitive Equations (CPEs). In this case, we say that the density $\rho_{\varepsilon}(t, x, y, z)$ is stratified with respect to the altitude $z$. It means that for each altitude $z$, the density is composed of "layers" $\rho_{0}(t, x)$. Taking advantage of this form, Ersoy and Ngom [10] show the existence of a global weak solution of CPEs. Moreover, such a structure lead to canonical averaging while the case $\gamma \neq 1$ will requires an extra assumption.

\section{Case $\gamma \neq 1$.}

At this stage, let us outline that the approximation

$$
\overline{\rho_{\varepsilon}^{\gamma}} \approx{\overline{\rho_{\varepsilon}}}^{\gamma}, \gamma \neq 1
$$

is obviously wrong except if $N=O(1)$, i.e. if the dimensionless Oser number is assumed small. Therefore, in what follows, we assume $C=O\left(\varepsilon^{l}\right)$ for some $l \geqslant \frac{1}{2}$. Thus, it yields to the following approximations

$$
\overline{\rho_{\varepsilon}}=\rho_{0}+O\left(C^{2}\right)=\rho_{0}+O\left(\varepsilon^{2 l}\right) \text { and } \overline{\rho_{\varepsilon}^{\gamma}}=\rho_{0}^{\gamma}+O\left(\varepsilon^{2 l}\right), \gamma \neq 1 \text {. }
$$

As a consequence, at first order, the approximation of the non linear terms are

$$
\overline{\rho_{\varepsilon} u_{\varepsilon}}=\overline{\rho_{\varepsilon} u_{\varepsilon}} \text { and } \overline{\rho_{\varepsilon}^{\gamma}}=\overline{\rho_{\varepsilon}} \gamma
$$

where

$$
\overline{\rho_{\varepsilon}}(t, x)=\frac{1}{S(x)} \int_{\Omega(x)} \rho_{\varepsilon}(t, x, y, z) d y d z .
$$




\section{Case $\gamma=1$.}

The smallness assumption on the Oser number is not required for linear pressure law since the density is stratified (see Equation (22) and Remark 3.1). Indeed, defining the weighted section $\mathcal{S}$ :

$$
\mathcal{S}(x)=\int_{\Omega(x)} \exp \left(-C^{2} \cos \theta(x) z\right) d y d z=\int_{-R(x)}^{R(x)} \exp \left(-C^{2} \cos \theta(x) z\right) \sigma(x, z) d z
$$

the averaged density reads:

$$
\overline{\rho_{\varepsilon}}(t, x)=\rho_{0}(t, x) \frac{\mathcal{S}(x)}{S(x)} .
$$

Thus, the non linear term $\overline{\rho_{\varepsilon} u_{\varepsilon}}$ can be approximated by

$$
\overline{\rho_{\varepsilon} u_{\varepsilon}}=\frac{1}{S} \int_{\Omega} \rho_{\varepsilon} u_{\varepsilon} d y d z \approx \rho_{0} \frac{\mathcal{S}}{S} \overline{u_{\varepsilon}}=\overline{\rho_{\varepsilon}} \overline{u_{\varepsilon}}
$$

and in particular, in view of (21) and (22), we get at first order

$$
\overline{\rho_{\varepsilon} u_{\varepsilon}^{2}}=\overline{\rho_{\varepsilon}}{\overline{u_{\varepsilon}}}^{2} \text {. }
$$

\subsection{Averaged pressurized model}

Let us first recall that $\mathbf{m}=(y, \varphi(x, y)) \in \partial \Omega(x)$ stands for the vector $\omega \mathbf{m}$ and $\mathbf{n}=\frac{\mathbf{m}}{|\mathbf{m}|}$ for the outward unit normal vector to the boundary $\partial \Omega(x)$ at the point $\mathbf{m}$ in the $\Omega$-plane (as displayed in figure $1(\mathrm{~b})$ ).

Then, gathering results (21), (23), (24) and (21), (26), (27), (28), integrating Equations (16)-(18) over the cross-section $\Omega$, we obtain

$$
\left\{\begin{aligned}
\partial_{t}\left(\overline{\rho_{\varepsilon}} S\right)+\partial_{x}\left(\overline{\rho_{\varepsilon}} S \overline{u_{\varepsilon}}\right) & =\int_{\partial \Omega(x)} \rho_{\varepsilon}\left(u_{\varepsilon} \partial_{x} \mathbf{m}-\boldsymbol{v}_{\varepsilon}\right) \cdot \mathbf{n} d s \\
\partial_{t}\left(\overline{\rho_{\varepsilon}} S \overline{u_{\varepsilon}}\right)+\partial_{x}\left(\overline{\rho_{\varepsilon}} S{\overline{u_{\varepsilon}}}^{2}+\frac{1}{M_{a}^{2}}{\overline{\rho_{\varepsilon}}}^{\gamma} S\right)= & \overline{\rho_{\varepsilon}} S \sin \theta(x) F_{L}^{2}+\frac{1}{M_{a}^{2}} \overline{\bar{\varepsilon}_{\varepsilon}} \gamma \frac{d S}{d x} \\
& +\int_{\partial \Omega(x)} \rho_{\varepsilon} u_{\varepsilon}\left(u_{\varepsilon} \partial_{x} \mathbf{m}-\mathbf{v}\right) \cdot \mathbf{n} d s \\
& -\int_{\partial \Omega(x)} \frac{\mu_{0}}{\varepsilon} \nabla_{y, z} u_{\varepsilon} \cdot \mathbf{n} d s
\end{aligned}\right.
$$

Next, using the no-penetration condition (8), the boundary integrals vanishes:

$$
\int_{\partial \Omega(x)} \rho_{\varepsilon}\left(u_{\varepsilon} \partial_{x} \mathbf{m}-\boldsymbol{v}_{\varepsilon}\right) \cdot \mathbf{n} d s=\int_{\partial \Omega(x)} \rho_{\varepsilon} u_{\varepsilon}\left(u_{\varepsilon} \partial_{x} \mathbf{m}-\boldsymbol{v}_{\varepsilon}\right) \cdot \mathbf{n} d s=0 .
$$

\section{Friction term.}

Using the approximations (21), (23), (24) and (21), (26), (27), (28), keeping in mind Equations (15) and (19), the reminding boundary integral becomes:

$$
\int_{\partial \Omega(x)} \frac{\mu_{0}}{\varepsilon} \nabla_{y, z} u_{\varepsilon} \cdot \mathbf{n} d s=\int_{\partial \Omega(x)} \rho_{\varepsilon} K_{0}\left(u_{\varepsilon}\right) d s=\rho_{0} \mathcal{S}_{\gamma}(x) K_{\gamma}\left(x, \overline{u_{\varepsilon}}\right)
$$

where

$$
K_{\gamma}\left(x, \overline{u_{\varepsilon}}\right)=K_{0}\left(\overline{u_{\varepsilon}}\right) \frac{s_{\gamma(x)}}{\mathcal{S}_{\gamma}(x)}
$$

with

$$
\mathcal{S}_{\gamma}(x)=\left\{\begin{array}{lll}
S(x) & \text { if } & \gamma \neq 1 \\
\mathcal{S}(x) & \text { if } & \gamma=1
\end{array}\right.
$$


In this expression, $s_{\gamma}(x)$ is the curvilinear integral of

$$
w_{\gamma, x}(z)=\left\{\begin{array}{lll}
1 & \text { if } \quad \gamma \neq 1 \\
\exp \left(-C^{2} \cos \theta(x) z\right) & \text { if } \quad \gamma=1
\end{array}\right.
$$

along the curve $\partial \Omega(x)$, i.e.,

$$
s_{\gamma}(x)=\int_{\partial \Omega(x)} w_{\gamma, x} d s=\int_{0}^{1} w_{x}(z(t)) \sqrt{\left(y^{\prime}(t)\right)^{2}+\left(z^{\prime}(t)\right)^{2}} d t
$$

where $t \in[0,1] \mapsto(y(t), z(t))$ stands for a parametrization of the curve $\partial \Omega(x)$. If $\gamma \neq 1, s_{\gamma}(x)$ corresponds to the wet perimeter of the section $\Omega(x)$ while $s_{1}(x)$ is the weighted wet perimeter of the section $\Omega(x)$.

\section{Remark 3.2.}

1. For practical situations, the pipe section is either rectangular, circular or "horseshoe" type. Therefore, the quantity $\mathcal{S}_{\gamma}(32)$ and $s_{\gamma}$ (33), appearing in the expression of the friction (31) for instance, can be explicitly computed. For example for a pipe with rectangular section $\Omega(x)=\left[-\frac{T(x)}{2}, \frac{T(x)}{2}\right] \times$ $[-R(x), R(x)]$ of width $\sigma(x, z)=T(x)$ and height $2 R(x)$, the weighted area for $\gamma=1$ is

$$
\mathcal{S}_{1}(x)=\mathcal{S}(x)=\frac{T(x)}{C^{2} \cos \theta(x)}\left(\exp \left(C^{2} \cos \theta(x) R\right)-\exp \left(-C^{2} \cos \theta(x) R\right)\right)
$$

and if $\gamma \neq 1$, then $\mathcal{S}_{\gamma}$ corresponds to the physical area, i.e., $\mathcal{S}_{\gamma}=S(x)=2 R(x) T(x)$. In the same manner, the weighted wet perimeter for $\gamma=1$ is

$$
s_{1}=\frac{2\left(\exp \left(C^{2} \cos \theta(x) R\right)-\exp \left(-C^{2} \cos \theta(x) R\right)\right)}{C^{2} \cos \theta(x)}+2 T(x)\left(\exp \left(C^{2} \cos \theta(x) R\right)+\exp \left(-C^{2} \cos \theta(x) R\right)\right)
$$

and if $\gamma \neq 1$ then $s_{\gamma}$ is the wet perimeter of the section $\Omega(x)$, i.e, $s_{\gamma}=4 R(x)+2 T(x)$.

2. For linear pressure law $(\gamma=1)$ and for low Oser flows $(C \ll 1)$, as one can notice in the above expressions for pipe with rectangular section, the weighted wet perimeter $\mathcal{S}_{1}=\mathcal{S}(25)$, (respectively $s_{1}$ (33)) corresponds approximately to the physical area (respectively the perimeter) of the pipe section $\Omega(x)$ as in the non linear case $(\gamma \neq 1)$.

3. For linear pressure law, we call the quantity $\left(\frac{\mathcal{S}_{1}(x)}{s_{1}(x)}\right)$ the weighted hydraulic radius. In particular, for low Oser flows, this quantity coincides with the classical hydraulic radius introduced by engineers as a length scale for non-circular ducts in similitude analysis (see for instance [21, 22]). Let us outline that this factor comes naturally with a weight depending on the Oser number. For the sake of completeness, we propose a numerical investigation of the influence of the Oser number for linear and non linear pressure laws in Section 4.

In view of Equations (23) and (26), thanks to the notation (32), we can write

$$
\overline{\rho_{\varepsilon}} S=\rho_{0} \mathcal{S}_{\gamma}
$$

so that, gathering Equations (29)-(34), the resulting system is

$$
\begin{cases}\partial_{t}\left(\rho_{0} \mathcal{S}_{\gamma}(x)\right)+\partial_{x}\left(\rho_{0} \mathcal{S}_{\gamma}(x) \overline{u_{\varepsilon}}\right) & =0, \\ \partial_{t}\left(\rho_{0} \mathcal{S}_{\gamma}(x) \overline{u_{\varepsilon}}\right)+\partial_{x}\left(\rho_{0} \mathcal{S}_{\gamma}(x){\overline{u_{\varepsilon}}}^{2}+\frac{1}{M_{a}^{2}} \rho_{0}^{\gamma} \mathcal{S}_{\gamma}(x)\right)= & \rho_{0} \mathcal{S}_{\gamma}(x) \sin \theta(x) F_{L}^{2}+\frac{1}{M_{a}^{2}} \rho_{0}^{\gamma} \mathcal{S}_{\gamma}(x) \frac{d \ln S}{d x}(x) \\ & -\rho_{0} \mathcal{S}_{\gamma}(x) K_{\gamma}\left(x, \overline{u_{\varepsilon}}\right) .\end{cases}
$$


Finally, multiplying Equations (35) by $\frac{r D U^{2}}{L}$ in order to recover the variables with dimension and skipping the index 0 for the averaged unknowns of density $\rho$ and fluid velocity $u$, defining the pressure by

$$
p_{\gamma}(x, \rho)=\alpha \rho^{\gamma} \mathcal{S}_{\gamma}(x)=\left\{\begin{array}{lll}
\alpha \rho^{\gamma} S(x) & \text { if } & \gamma \neq 1 \\
\alpha \rho \mathcal{S}(x) & \text { if } & \gamma=1
\end{array}\right.
$$

we get the $\gamma$-pressurized model:

$$
\begin{cases}\partial_{t}\left(\rho \mathcal{S}_{\gamma}(x)\right)+\partial_{x}\left(\rho \mathcal{S}_{\gamma}(x) u\right) & =0, \\ \partial_{t}\left(\rho \mathcal{S}_{\gamma}(x) u\right)+\partial_{x}\left(\rho \mathcal{S}_{\gamma}(x) u^{2}+p_{\gamma}(x, \rho)\right)= & g \rho \mathcal{S}_{\gamma}(x) \sin \theta(x)+p_{\gamma}(x, \rho) \frac{d \ln S}{d x}(x) \\ & -\rho \mathcal{S}_{\gamma}(x) K_{\gamma}(x, u)\end{cases}
$$

where the source terms are

- $g \rho \mathcal{S}_{\gamma}(x) \sin \theta(x)$ often noted $-g \rho \mathcal{S}_{\gamma}(x) \frac{d Z}{d x}(x)$ is the altitude of the main pipe axis,

- $\alpha \rho^{\gamma} \mathcal{S}_{\gamma}(x) \frac{d \ln S}{d x}(x)$ is the pressure source term which takes into account the change of section, and

- $-\rho \mathcal{S}_{\gamma}(x) K_{\gamma}(x, u)$ is the friction term where $K_{\gamma}$ is defined by Equation (31).

Remark 3.3. Bourdarias et al. $[1,4,5]$ has proposed a Finite Volume Kinetic scheme for the 1-pressurized and $\frac{7}{5}$-pressurized model in the context of water and air flows in closed pipes. Through several numerical experiments versus laboratory tests, the numerical model has been validated. Let us also mention that, following [4], the kinetic scheme can be easily adapted to the $\gamma$-pressurized model.

Let us define the following quantities:

- $c_{\gamma}^{2}(x, \rho)=\frac{\partial p_{\gamma}}{\partial \rho}(x, \rho)$,

- $P_{\gamma}(x, \rho)=\left\{\begin{array}{lll}\alpha \frac{\gamma}{\gamma-1} \rho^{\gamma-1} & \text { if } & \gamma \neq 1 \\ \alpha \ln \left(\rho \frac{\mathcal{S}_{\gamma}(x)}{S(x)}\right) & \text { if } & \gamma=1\end{array}\right.$

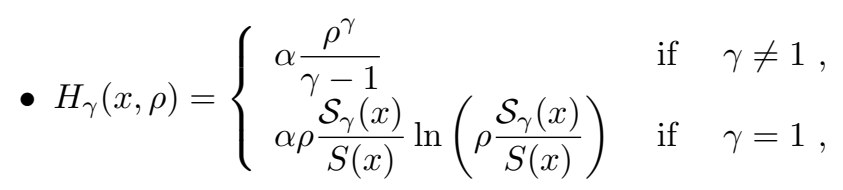

Then, System (37) has the following properties:

\section{Theorem 3.1.}

1. System (37) is strictly hyperbolic on $\{\rho(t, x)>0\}$.

2. For smooth solutions, the mean velocity u satisfies:

$$
\partial_{t} u+\partial_{x}\left(\frac{u^{2}}{2}+P_{\gamma}(x, \rho)+g Z(x)\right)=-K_{\gamma}(x, u) .
$$

The quantity $\frac{u^{2}}{2}+P_{\gamma}(x, \rho)+g Z(x)$ is the so-called the total head. 
3. For smooth solutions, the still steady state, i.e. $u=0$, for System (37) is:

$$
P_{\gamma}(x, \rho)+g Z(x)=c t e
$$

for some constant cte.

4. System (37) admits a mathematical entropy:

$$
E_{\gamma}(x, \rho, u)=\frac{\rho u^{2}}{2}+H_{\gamma}(x, \rho)+g \rho Z(x)
$$

which satisfies the entropy relation for smooth solutions

$$
\partial_{t} E_{\gamma}(x, \rho, u)+\partial_{x}\left(\left(E_{\gamma}(x, \rho, u)+p_{\gamma}(x, \rho)\right) u\right)=-\rho u K_{\gamma}(x, u) \leqslant 0
$$

Proof. The proof of these assumptions relies only on algebraic combinations of the two equations of System (37) and is left to the reader.

\section{The Oser number and a formal second order approximation}

In Section 3, we have constructed the $\gamma$-pressurized model (37) from the Navier-Stokes equations (1). In particular, we have introduced the Oser number defined as $C=M_{a} / F_{r}$ where $M_{a}, F_{r}$ are respectively the Mach and the Froude number. The Oser number is a dimensionless number which measures the influence of the gravity on the flow. For $\gamma=1$, the Oser number appears directly into the friction term (31) as a "weighted" measure through the terms (32) and (33) and therefore plays a role in the second order approximation. Let us recall that, for $\gamma \neq 1$, the approximation $\overline{\rho^{\gamma}}$ by $\bar{\rho}^{\gamma}$ holds true if the Oser number is assumed small.

For the sake of completeness, we propose to numerically study the influence of the Oser number in the case of pipe with circular section. Keeping in mind the asymptotic assumptions (14), let us first consider the equation of the conservation of the momentum (10)

$$
\begin{aligned}
& \operatorname{div}_{y, z}\left(\frac{\mu}{\varepsilon} \nabla_{y, z} u_{\varepsilon}\right)=\partial_{t}\left(\rho_{\varepsilon} u_{\varepsilon}\right)+\partial_{x}\left(\rho_{\varepsilon} u_{\varepsilon}^{2}\right)+\operatorname{div}_{y, z}\left(\rho_{\varepsilon} u_{\varepsilon} \boldsymbol{v}_{\varepsilon}\right)+\frac{1}{M_{a}^{2}} \partial_{x} \rho_{\varepsilon}^{\gamma}+\rho_{\varepsilon} \frac{\sin \theta(x)}{F_{L}^{2}} \\
& =\left\{\begin{array}{lll}
\rho_{\varepsilon}\left(\partial_{t}\left(u_{\varepsilon}\right)+\mathbf{u}_{\varepsilon} \cdot \nabla\left(u_{\varepsilon}\right)+\frac{1}{M_{a}^{2}} \partial_{x} \ln \left(\rho_{\varepsilon}\right)+\frac{\sin \theta(x)}{F_{L}^{2}}\right) & \text { if } & \gamma=1 \\
\rho_{\varepsilon}\left(\partial_{t}\left(u_{\varepsilon}\right)+\mathbf{u}_{\varepsilon} \cdot \nabla\left(u_{\varepsilon}\right)+\frac{\gamma}{M_{a}^{2}} \rho_{\varepsilon}^{\gamma-2} \partial_{x} \rho_{\varepsilon}+\rho_{\varepsilon} \frac{\sin \theta(x)}{F_{L}^{2}}\right) & \text { if } & \gamma \neq 1
\end{array}\right.
\end{aligned}
$$

Next using the motion by slices "property" (20) and the total head equation (38) on the right hand side of the above equations, writing $\frac{d Z}{d x}(x)=-\sin (\theta(x))$, we get for all $x \in(0, L)$ and $(y, z) \in \Omega(x)$,

$$
\operatorname{div}_{y, z}\left(\frac{\mu}{\varepsilon} \nabla_{y, z} u_{\varepsilon}\right)=-\rho_{\varepsilon} K_{\gamma}(x, u)+O(\varepsilon)
$$

where $\rho_{\varepsilon}$ is approximated by (22) and $K_{\gamma}$ is given by (31). In view of the boundary conditions (19), the solution of the problem (39) yields to the second order "paraboloid" approximation of $u_{\varepsilon}$. This approximation depends on the Oser number through the density $\rho_{\varepsilon}(22)$ and the friction term (31).

To simplify the numerical problem, we assume $\rho_{0}=1$ in (22), $u_{0}=1$ in $(20), \mu=1$ and we fix $\varepsilon=10^{-3}$. Moreover, we assume the friction factors $C_{l}$ and $C_{t}$ are such that $K_{0}(x, u)=1$ (see Equations (31), (15) and (5)). Then, we compute for several value of $C$ and $\gamma$ the velocity profile for a circular pipe section of radius 1.

As expected, for small Oser number, the influence of the gravity can be neglected. We illustrate this statement for $\gamma=1,1.4,2$ and 5 and we observe that the paraboloid profile is well-centred (see figures 3(a), 
3(b), 3(c) and 3(d)). Moreover, there are no significant changes between linear and non linear results since the right hand side of Equation (39) is almost equal. Indeed, for linear and non linear pressure law, in view of Equation (22), one has $\rho_{\varepsilon} \approx 1$ and as noticed in Remark 3.2, for small Oser number the expression of the friction term (31) is almost the same.

In figure 4, for linear pressure law $(\gamma=1)$, we display the velocity profile for $C=1, C=2, C=2.5$ and $C=3$ to show the influence of the Oser number. In these cases the effect of the gravity cannot be neglected and we observe that the velocity profiles are not longer centred when compared to the result in figure 3(a). In particular, the more the Oser number $C$ is large and the more the gravity plays an important role as illustrated in figures 4(a), 4(b), 4(c) and 4(d).

The results for the non linear case $(\gamma \neq 1)$ are not displayed since the $\gamma$-pressurized model is no longer valid for this range of value.

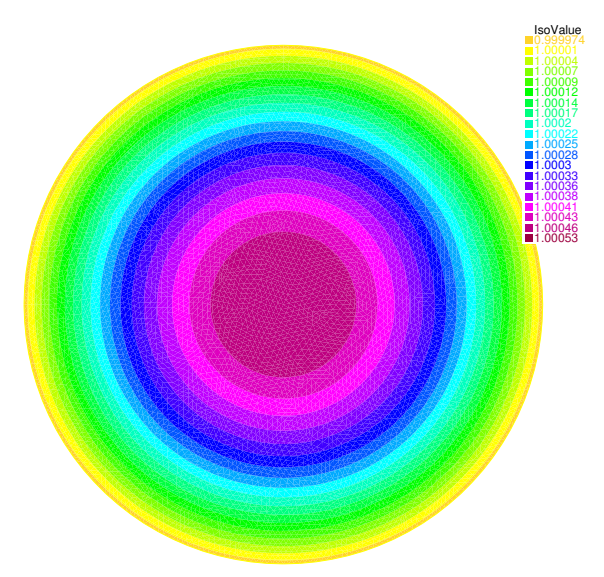

(a) $\gamma=1$

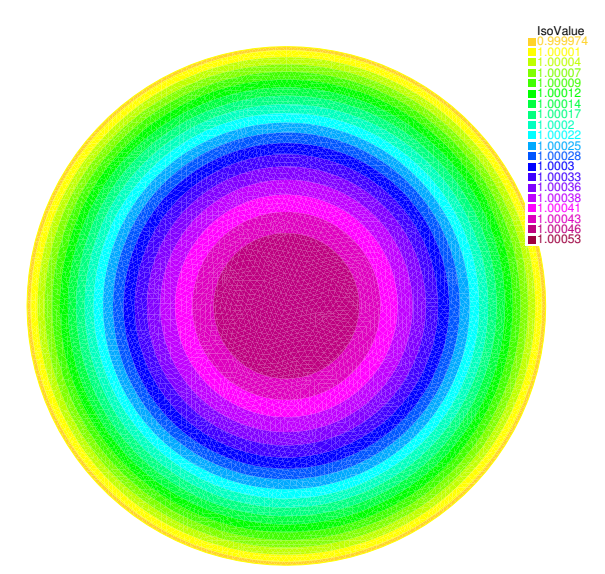

(c) $\gamma=2$

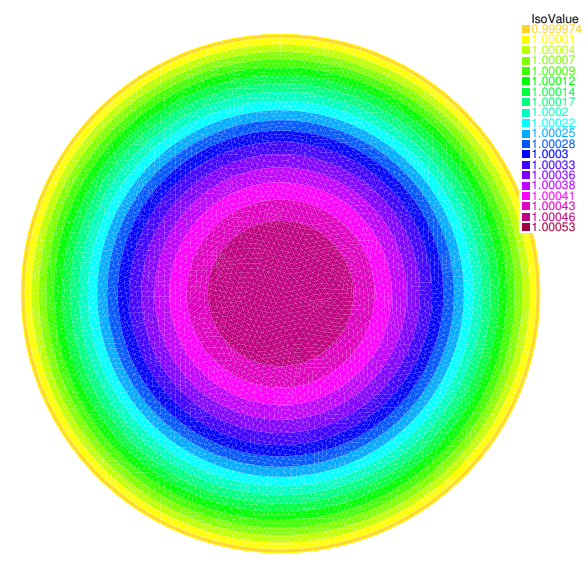

(b) $\gamma=1.4$

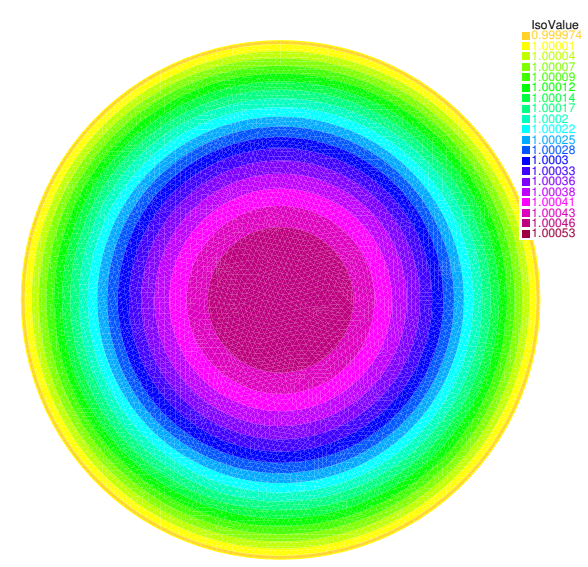

(d) $\gamma=5$

Figure 3: Velocity profile for $C=1 e-05$ 


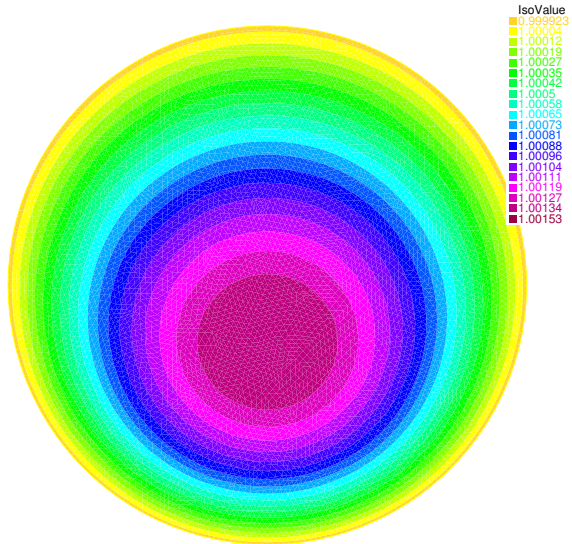

(a) $C=1$

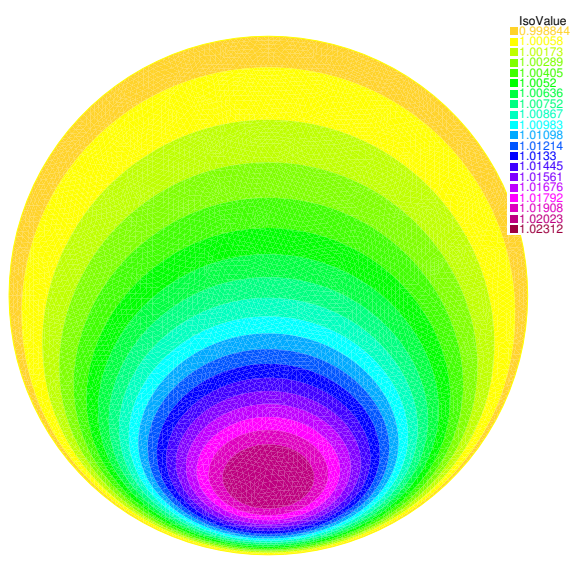

(c) $C=2.5$

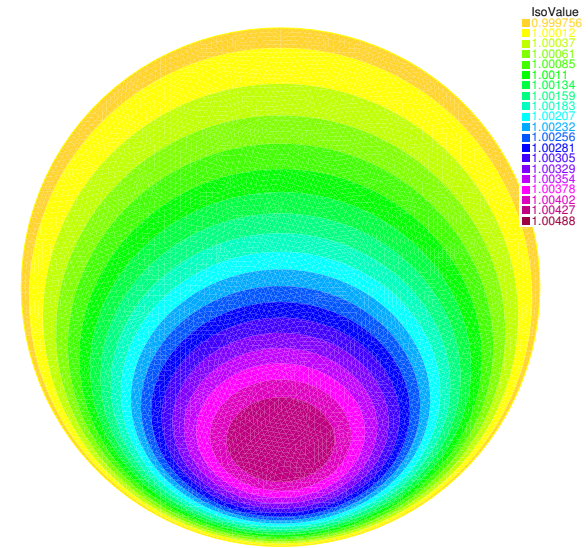

(b) $C=2$

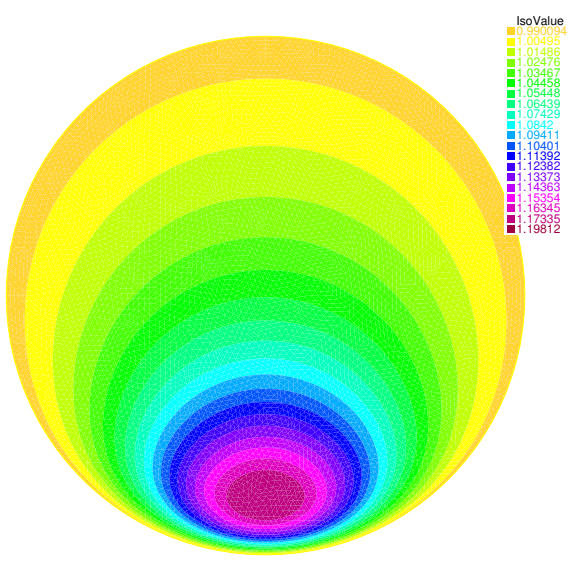

(d) $C=3$

Figure 4: Velocity profile for large Oser number for linear pressure law.

\section{Conclusions}

We have presented the derivation of a one dimensional barotropic compressible system of equations called $\gamma$-pressurized model including the friction. The hyperbolic $\gamma$-pressurized model (37) is, formally, an approximation of $O(\varepsilon)$ of the hydrostatic approximation (16)-(18) and therefore of the compressible Navier-Stokes equations (1). Unlike previous works by Bourdarias et al $[3,4]$, we have proposed a full justification (derivation) of the $\mathbf{P}$-model and the air entrainment model. To this purpose, first, we have obtained the "motion by slices" property required in the averaging process. Second, considering viscous flows instead of inviscid one, we have integrated the border friction in the model reduction. In particular, we have explicitly described the geometrical dependency (with respect to the hydraulic radius) and the hydrodynamical dependency (with respect to the gravity force through the Oser number) of the "averaged" friction appearing in the $\gamma$-pressurized model. 


\section{Acknowledgements}

This work is supported by the ModTerCom project within the APEX program of the region Provence-AlpeCôte d'Azur and the Project MTM2011-29306-C01-01 from the MICINN (Spain). The author thanks the referees for their valuable remarks which led to substantial improvement of the first version of this paper.

\section{References}

[1] C. Bourdarias, M. Ersoy, and S. Gerbi. A kinetic scheme for pressurised flows in non uniform closed water pipes. In Maths and water, Monogr. Real Acad. Ci. Exact. Fís.-Quím. Nat. Zaragoza, 31, pages 1-20. Real Acad. Ci. Exact., Fís. Quím. Nat. Zar, Zaragoza, 2009.

[2] C. Bourdarias, M. Ersoy, and S. Gerbi. A model for unsteady mixed flows in non uniform closed water pipes and a well-balanced finite volume scheme. Int. J. Finite Vol., 6(2):47, 2009.

[3] C. Bourdarias, M. Ersoy, and S. Gerbi. A mathematical model for unsteady mixed flows in closed water pipes. Sci. China Math., 55(2):221-244, 2012.

[4] C. Bourdarias, M. Ersoy, and S. Gerbi. Air entrainment in transient flows in closed water pipes: a two-layer approach. ESAIM: M2AN, 47(2):507-538, 2013.

[5] C. Bourdarias, M. Ersoy, and S. Gerbi. A model for unsteady mixed flows in non uniform closed water pipes: a Full Kinetic Approach. Numer. Math., 128(2):217-263, 2014.

[6] H. Capart, X. Sillen, and Y. Zech. Numerical and experimental water transients in sewer pipes. J. of Hydr. Res., 35(5):659-672, 1997.

[7] N. T. Dong. Sur une méthode numérique de calcul des écoulements non permanents soit à surface libre, soit en charge, soit partiellement à surface libre et partiellement en charge. La Houille Blanche, 2:149-158, 1990.

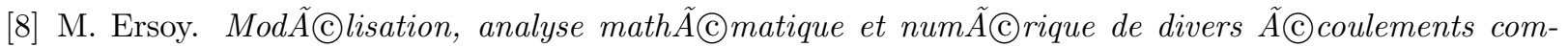
pressibles ou incompressibles en couche mince. PhD thesis, Université de Savoie, 2010. available at http://tel.archives-ouvertes.fr/tel-00529392.

[9] M. Ersoy. Dimension reduction for incompressible pipe and open channel flow including friction. In J. Brandts, S. Korotov, M. Krizek, K. Segeth, J. Sistek, and T. Vejchodsky, editors, Conference Applications of Mathematics 2015, in honor of the birthday anniversaries of Ivo Babuska (90), Milan Prager (85), and Emil Vitasek (85). Institute of Mathematics CAS, 2015.

[10] M. Ersoy and T. Ngom. Existence of a global weak solution to compressible primitive equations. C. $R$. Math. Acad. Sci. Paris, 350(7-8):379-382, 2012.

[11] M. Ersoy, T. Ngom, and M. Sy. Compressible primitive equations: formal derivation and stability of weak solutions. Nonlinearity, 24(1):79-96, 2011.

[12] M. Fuamba. Contribution on transient flow modelling in storm sewers. J. of Hydr. Res., 40(6):685-693, 2002.

[13] P. Garcia-Navarro, F. Alcrudo, and A. Priestley. An implicit method for water flow modelling in channels and pipes. J. of Hydr. Res., 32(5):721-742, 1994.

[14] J.-F. Gerbeau and B. Perthame. Derivation of viscous Saint-Venant system for laminar shallow water; numerical validation. Discrete Cont. Dyn. Syst. Ser. B, 1(1):89-102, 2001. 
[15] M. Hamam and A. McCorquodale. Transient conditions in the transition from gravity to surcharged sewer flow. Can. J. Civ. Eng., 9:189-196, 1982.

[16] N. E. Kochin. On simplification of the equations of hydromechanics in the case of the general circulation of the atmosphere. Trudy Glavn. Geofiz. Observator., 4:21-45, 1936.

[17] L. D. Landau and E. M. Lifshitz. Fluid mechanics. Translated from the Russian by J. B. Sykes and W. H. Reid. Course of Theoretical Physics, Vol. 6. Pergamon Press, London, 1959.

[18] T. Morales de Luna. A Saint Venant model for gravity driven shallow water flows with variable density and compressibility effects. Math. Comput. Modelling, 47(3-4):436-444, 2008.

[19] P. H. Oosthuizen and W. E. Carscallen. Compressible fluid flow. McGraw-Hill, New York, 1997.

[20] C. Song, J. Cardle, and K. Leung. Transient mixed-flow models for storm sewers. Journal of Hydraulic Engineering, ASCE, 109(11):1487-1503, 1983.

[21] V. Streeter and E. Wylie. Fluid Transients. McGraw-Hill, New York, 1978.

[22] V. Streeter and E. Wylie. Fluid transients in systems. Prentice Hall, Englewood Cliffs, NJ, 1993.

[23] V. Streeter, E. Wylie, and K. Bedford. Fluid Mechanics. McGraw-Hill, 1998.

[24] D. Wiggert. Transient flow in free surface, pressurized systems. Journal of the Hydraulics division, 98(1):11-27, 1972. 